\title{
Landau level crossing in a spin-orbit coupled two-dimensional electron gas
}

\author{
Xing-Jun $\mathrm{Wu},{ }^{1,2}$ Ting-Xin $\mathrm{Li}^{1,}{ }^{1,2}$ Chi Zhang, ${ }^{1,2}$ and Rui-Rui $\mathrm{Du}^{1,2,3}$ \\ ${ }^{1}$ International Center for Quantum Materials, School of Physics, Peking University, Beijing 100871, \\ People's Republic of China \\ ${ }^{2}$ Collaborative Innovation Center of Quantum Matter, Beijing 100871, People's Republic of China \\ ${ }^{3}$ Rice University, Houston, Texas 77251-1892, USA
}

(Received 6 December 2014; accepted 22 December 2014; published online 6 January 2015)

\begin{abstract}
We have studied experimentally the Landau level (LL) spectrum of a two-dimensional electron gas (2DEG) in an $\mathrm{In}_{0.53} \mathrm{Ga}_{0.47} \mathrm{As} / \mathrm{InP}$ quantum well structure by means of low-temperature magnetotransport coincidence measurement in vector magnetic fields. It is well known that LL crossing occurs in tilted magnetic fields due to a competition between cyclotron energy and Zeeman effect. Remarkably, here we observe an additional type of level-crossing resulting from a competition between Rashba and Zeeman splitting in a small magnetic field, consistent with the theoretical prediction for strongly spin-orbit coupled 2DEG. (C) 2015 AIP Publishing LLC.
\end{abstract}

[http://dx.doi.org/10.1063/1.4905492]

In recent years, InAs-based heterostructures like InAs/ $\mathrm{AlSb}$ and InGaAs/InAlAs have been actively studied ${ }^{1-3}$ both experimentally and theoretically, motivated by a better understanding of the rich physical phenomena related to strong spin-orbit (SO) coupling in these quantum structures. Devices based on strongly SO-coupled two-dimension electron gas (2DEG) have potential applications in spin manipulation and quantum information processing, because spin properties such as effective g-factor or spin-polarization can be electrically controlled. Such properties can often be measured by magneto-transport under tilted magnet fields. ${ }^{4,5}$ It is well known that for a $2 \mathrm{DEG}$, the cyclotron energy $\mathrm{E}_{\mathrm{C}}$ is proportional to the perpendicular magnetic field while the Zeeman energy $E_{Z}$ is proportional to the total field. By increasing the in-plane field $\mathrm{B}_{/ /}, \mathrm{E}_{\mathrm{Z}}$ increases while $\mathrm{E}_{\mathrm{C}}$ remains constant, leading to a transition between the adjacent spin-split Landau levels (LL) from spin-unpolarized state to a partially spin-polarized state.

Generally in a single-electron picture, the coincidence of LLs between different orbits should happen for integer ratio of $\mathrm{E}_{\mathrm{Z}} / \mathrm{E}_{\mathrm{C}}$. However, due to exchange interaction, the LL spectrum is strongly modified from single particle picture. For example, enhanced g-factor ${ }^{5,6}$ or anti-crossings of $\mathrm{LLs}^{7}$ have be observed experimentally. Additionally, zero-field spin splitting due to the existence of strong SO (especially Rashba term ${ }^{8}$ ) has been studied ${ }^{9-11}$ experimentally. Weak anti-localization $^{12,13}$ or pronounced beating pattern ${ }^{13-15}$ in Shubnikov de Haas $(\mathrm{SdH})$ oscillations has been primary tools in measuring the zero-field spin splitting in 2DEG. Landau level crossing could occur even in a small magnetic field due to the competition between Rashba and Zeeman splitting, where spin-splitting gap should close before reopening in sweeping the field. ${ }^{16-18}$ We report here magneto-resistance evidence that such LL crossing occurs in a strongly SOcoupled 2DEG. It has been theoretically predicted that a "resonant spin Hall effect" should arise at such levelcrossing, resulting in polarized spin currents that can be controlled by electrostatic gates. ${ }^{16-18}$ Our experiment results indicate that such condition could be reached in a conventional semiconductor quantum well (QW).
In this paper, we present results of level coincidence spectrum in $\mathrm{In}_{0.53} \mathrm{Ga}_{0.47} \mathrm{As} / \mathrm{InP} \mathrm{QW}$ subjected to a vector magnetic field, where the perpendicular field $\mathrm{B}_{\perp}$ and the parallel field $\mathrm{B}_{/ /}$were provided independently from two superconductor coils and effective tilt angle $\theta=\tan ^{-1}\left(\mathrm{~B}_{/ /} / \mathrm{B}_{\perp}\right)$. The data were analyzed based on a simple model of LL degeneracy, from which we can delineate three different spin-splitting mechanisms: Rashba spin-orbit coupling, bare Zeeman effect, and exchange-interaction terms. Rashba term dominates in the small tilted field regime. With increasing field or tilt angle, the main spin-splitting mechanisms are Zeeman and exchange terms. Several main features should be highlighted before the detailed descriptions. (1) We resolve step-like level spectrum for the LL filling factor as high as $\nu \cong 20$, which implies the presence of transitions between spin-unpolarized and spin-polarized states. (2) Remarkably, we observe additional level crossing in small tilt angles resulting from the opposite signs of Rashba and the Zeeman terms, which has not been reported until this work. And (3), we observe an enhanced $g$-factor, $g^{*}$, which underscores the contributions from exchange interaction.

The sample used in this study is an unintentional-doped $\mathrm{In}_{0.53} \mathrm{Ga}_{0.47} \mathrm{As} / \mathrm{InP} \mathrm{QW}$. The structure is grown by metal organic chemical vapor deposition (MOCVD) on an InP substrate, consisting of a $150 \mathrm{~nm} \mathrm{InP} \mathrm{buffer} \mathrm{layer,} \mathrm{a} 500 \mathrm{~nm}$ $\mathrm{In}_{0.53} \mathrm{Ga}_{0.47}$ As layer lattice matched to InP, and a $5 \mathrm{~nm} \mathrm{InP}$ cap layer. As depicted in the inset of Fig. 1, the 2DEG is formed near the bottom interface between $\mathrm{In}_{0.53} \mathrm{Ga}_{0.47} \mathrm{As}$ and InP. The sample was processed into a standard Hall bar with the size of $0.1 \times 0.5 \mathrm{~mm}^{2}$ and the measurement was performed in a top-loading He-3 cryostat (base temperature of $300 \mathrm{mK}$ ) equipped with a two-axis superconducting magnet of $9 \mathrm{~T}(4 \mathrm{~T})$ vertical (horizontal) maximum field. Fourterminal magneto-resistance $R_{x x}$ and Hall resistance $R_{x y}$ were measured using standard low frequency $(17 \mathrm{~Hz})$ lock-in techniques with a $100-n A$ bias current. The 2 DEG is characterized by a carrier density of $\mathrm{n}_{\mathrm{s}}=6 \times 10^{10} / \mathrm{cm}^{2}$, calculated from $\mathrm{R}_{\mathrm{xy}}$, and a mobility of $\mu=1 \times 10^{5} \mathrm{~cm}^{2} / \mathrm{V} \cdot \mathrm{s}$.

The electron effective mass $\mathrm{m}^{*}$ in $\mathrm{In}_{0.53} \mathrm{Ga}_{0.47} \mathrm{As} / \mathrm{InP}$ quantum well structure was determined from $\mathrm{SdH}$ analysis 


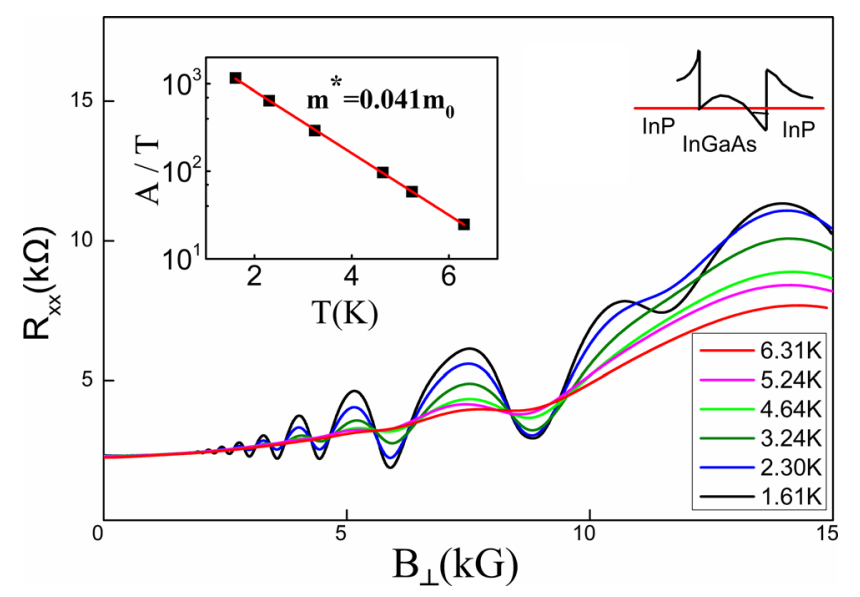

FIG. 1. Longitudinal resistance $\mathrm{R}_{\mathrm{xx}}$ versus perpendicular magnetic field for different temperatures. The left upper inset demonstrates the corresponding Dingle plot of the $\mathrm{SdH}$ amplitude A divided by the temperature T vs. the temperature variance $T$. The slope of the linear fit indicates $\mathrm{m}^{*}=0.041 \mathrm{~m}_{0}$.

of temperature-dependent magneto-resistance. As is well known, in small magnetic field, the $\mathrm{SdH}$ oscillations in resistance $R_{x x}$ can be described by the following equation: ${ }^{19,20}$

$$
\Delta R_{x x}=4 R_{0} \frac{X_{T}}{\sinh \left(X_{T}\right)} \exp \left(-\frac{D_{q}}{\hbar \omega_{c}}\right) \cos \left(\frac{2 \pi E_{F}}{\hbar \omega_{c}}-\pi\right),
$$

where $R_{0}$ is the zero field resistivity, $D_{q}=\pi \hbar / \tau_{q}$ is the Dingle damping parameter, $\tau_{\mathrm{q}}$ is the quantum lifetime, $\mathrm{E}_{\mathrm{F}}$ is electron Fermi energy, and $X_{T}=2 \pi^{2} k_{B} T / \hbar \omega_{c}$ with $k_{B}$ being the Boltzmann constant and $\hbar \omega_{\mathrm{c}}$ is the cyclotron energy. The 2DEG effective mass $\mathrm{m}^{*}$ can thus be extracted from the temperature- dependent $\mathrm{SdH}$ oscillation amplitude $\mathrm{A}(\mathrm{T})$, where $\mathrm{C}$ is a constant

$$
\ln (\mathrm{A} / \mathrm{T}) \cong \mathrm{C}-2 \pi^{2} \mathrm{k}_{\mathrm{B}} \mathrm{m}^{*} / \mathrm{e} \hbar \mathrm{BT} .
$$

Figure 1 displays $\mathrm{SdH}$ traces at different temperatures obtained by sweeping the perpendicular magnetic field, and the up-left inset shows the corresponding Dingle plot. The linear fit in the inset confirms the validity of Eq. (2), which gives $\mathrm{m}^{*} \cong 0.041 \mathrm{~m}_{0}$, where $\mathrm{m}_{0}$ is the free electron mass. The result is broadly consistent with the experiment value $\mathrm{m}^{*}=0.044 \mathrm{~m}_{0}$ previously reported for $\mathrm{In}_{0.53} \mathrm{Ga}_{0.47} \mathrm{As}{ }^{21}$ Comparing the quantum life time $\left(\tau_{\mathrm{q}}=0.95 \mathrm{ps}\right)$ from the Dingle plot with the Drude transport lifetime $\left(\tau_{\mathrm{c}}=2.3 \mathrm{ps}\right)$, estimated from mobility, we find that short-range scattering (including the interface roughness) mechanisms dominate the low-field transport, ${ }^{22}$ since the ratio $\left(\tau_{\mathrm{c}} / \tau_{\mathrm{q}}=2.4\right)$ is rather small.

Fig. 2 displays the longitudinal resistance $R_{x x}$ versus the perpendicular magnetic field $\mathrm{B}_{\perp}$ for different in-plane magnetic field $\mathrm{B}_{/ /}$. Several transitions between spin-split LLs can be observed at odd and even filling factors as shown in Fig. 2. Take $\nu=6$, for example, with increasing $\mathrm{B}_{/ /}$, positions of the two distinct peaks around $\nu=6$ (lying at $\mathrm{B} \approx 5.4 \mathrm{kG}$ and $3.9 \mathrm{kG}$, at zero in-plane magnetic field) become increasingly closer, eventually merge into a single peak around $\mathrm{B}_{/ /}=27 \mathrm{kG}$. We mark this point as $\nu=6, \ell=1$ as shown in the up-right part of Fig. 2, where $\nu$ is the corresponding filling factor and $\ell$ represents the ratio $\ell=\mathrm{E}_{\mathrm{z}} / \mathrm{E}_{\mathrm{c}}$,

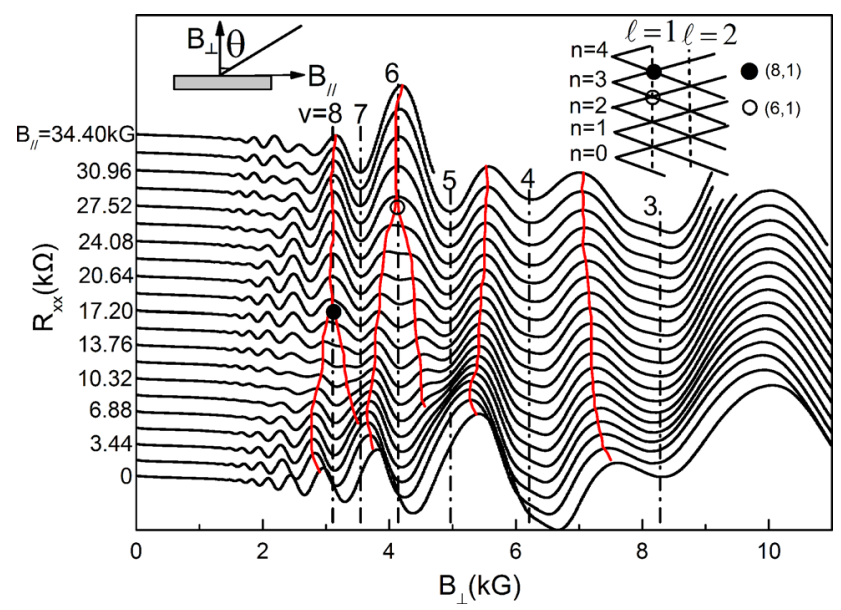

FIG. 2. Longitudinal resistance as a function of the perpendicular magnetic field for different horizontal magnetic fields measured at $\mathrm{T}=300 \mathrm{mK}$. Traces have been shifted vertically for clarity. Vertical thin dotted lines represent the integer filling factors. The red curves are plotted to emphasize the behavior of the oscillation patterns, and the solid and open circles in the figure describe the crossings at $(8,1)$ and $(6,1)$, respectively, which are also marked in corresponding positions in the upper-right inset. The upper-right inset: schematic diagram of the general spin-split Landau levels vs $1 / \cos \theta$, where LLs are marked by quantum number $n$. The up-left inset: sketch of the experimental geometry.

respectively. These coincidences occur at an integer ratio ( $\ell=1,2, \ldots$, etc.) between the exchange-enhanced Zeeman energy $E_{Z}$ and the cyclotron energy $E_{c}$. A similar scenario holds at $(8,1)$ point.

Since $E_{Z}$ is related to the total magnetic field $B$ while cyclotron energy $E_{C}$ depends only on $B_{\perp}$, the spin-split $L L$ coincidence is $\theta$-dependent, where tilt angle $\theta$ is defined in Fig. 2, and $\mathrm{B}_{\perp}=\mathrm{B} \cos \theta, \mathrm{B}_{/ /}=\mathrm{B} \sin \theta$. From Fig. 2, we extract every peak in $R_{x x}$, each corresponding to a pair of known $B_{\perp}$ and $\mathrm{B}_{/ /}$, or in other words, corresponding to an unique angle $\theta=\tan ^{-1}\left(\mathrm{~B}_{/ /} / \mathrm{B}_{\perp}\right)$. Mapping the peaks in each filling factor gives us a way to analysis the spin-split LL spectrum as a function of the tilt angles.

Fig. 3 shows the perpendicular magnetic field position of the $\mathrm{SdH}$ peaks as a function of $1 / \cos \theta$. It is clear that when increasing the tilt angle, the adjacent spin-split LLs come closer, and coincidence first occurs at even filling factors, such as $\nu=6,8$. In higher even filling factors, level coincidences are not directly observed due to the fact that fine details of the LL spectrum are not resolved at the measurement temperature of $300 \mathrm{mK}$. Nevertheless, we have observed step-like jumps appearing at higher filling factors, where every step-like jump corresponding to a transition between a spin-unpolarized state and a partially spinpolarized state. This convincingly demonstrates the coincidences of spin-split LLs in a wide range of filling factors.

With increasing tilt angle, LL crossing occurs consecutively: The Nth Landau level first crosses the $(\mathrm{N}+1)$ th $\mathrm{LL}$ at even filling factor, then with $(\mathrm{N}+2)$ th $\mathrm{LL}$ at odd, and so on. We can roughly divide our observed level-crossing phenomena into two groups. (1) for most of the first transitions $(\ell=1, \nu=6,8,10, \ldots$, etc. $)$, the perpendicular field $\mathrm{B}_{\perp}$ of the observed resistance peaks matches the corresponding filling factors well; (2) for the second transitions $(\ell=2, \nu=11,13$, etc.), the $B_{\perp}$ values of peaks do not exactly match the 


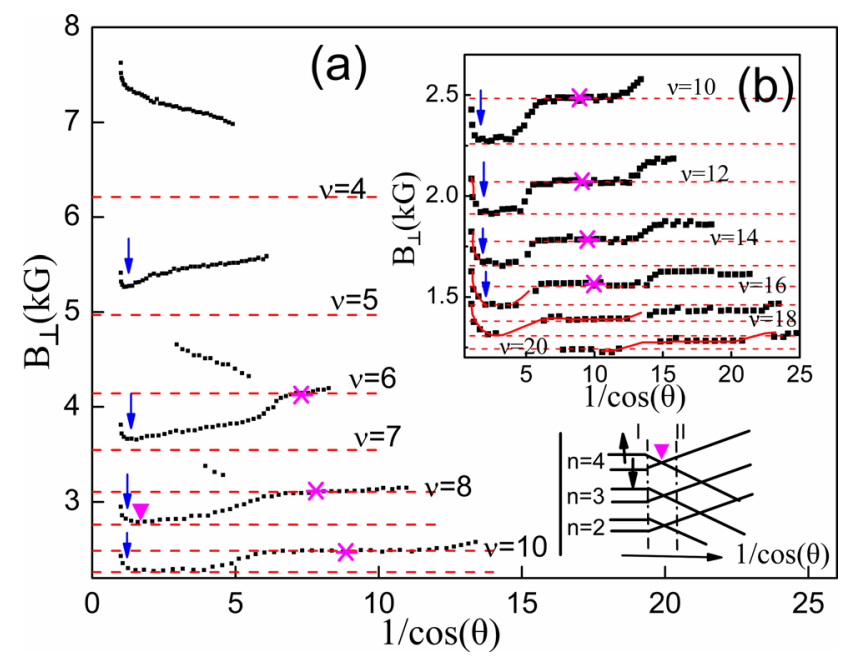

FIG. 3. Figures (a) and (b) describe the perpendicular magnetic field position of the longitudinal resistance peaks as a function of $1 / \cos \theta$ at low filling factor $(\nu \leq 10)$, and high filling factor $(\nu \geq 10)$, respectively. The thin red lines have been added to guide the eye, stressing the behavior of Landau levels at high filling factors, and blue arrows mark the anomalous behavior revealing the zero-field spin-splitting due to the Rashba term. The Rashba term is the dominant spin-splitting mechanism at small tilt angle regime (roughly, in the left regime of line I), whereas Zeeman term is dominant at large tilt angle regime (the right regime of line II). In the transition region, additional degeneracy occurs. The lower-right inset: schematic diagram of the spin-split Landau levels vs $1 / \cos \theta$ taking zero-field spin splitting into account, where LLs are marked by quantum number $\mathrm{n}$.

corresponding filling factors, for example, the peak position deviates significantly at $\nu=13$. The group (1) can be interpreted as a result of regular LL crossing, or, more specifically, the gap closes before it reopens. On the other hand, the observations for the group (2) suggest that the gap does not fully close before it reopens. Such anti-crossing could happen due to spin-orbit interaction and nonparabolicity (NP) of the band structures, as suggested by Desrat et al. ${ }^{7}$ Unlike the transitions at $\ell=1$ that are governed mainly by the k-linear terms (Rashba term here) to couple adjacent Landau levels, the transitions at $\ell=2$ are due to NP terms. The NP terms cannot be neglected due to the large magnetic field and become more significant in our system to induce the observed anti-crossings; similar behavior could be found in Ref. 7.

In order to further delineate Zeeman splitting term and the exchange-correlation term, we extract the bare g-factor from the experimental data shown in Fig. 3. In principle, from coincidence condition, exchange-enhanced g-factor can be determined as $\left|\mathrm{g}^{*}\right|=2 \mathrm{~m}_{0} \cos \theta_{\mathrm{c}} / \mathrm{m}^{*},{ }^{23}$ where $\theta_{\mathrm{c}}$ is a critical angle at which the gap completely closes. However, due to level broadening, it is difficult to accurately determine $\theta_{\mathrm{c}}$ from experiment. Here, we simply take the center point (marked by stars) of the plateau where coincidence takes place, to calculate the $\theta_{\mathrm{c}}$.

Figure 4 displays the perpendicular magnetic field dependence of the exchange-enhanced g-factor, which is approximately linear with $\mathrm{B}_{\perp}$. Empirically, we thus obtain a bare g-factor $\mathrm{g}_{0} \sim 3.9$, which is consistent with $|\mathrm{g}|=4.1$ from ESR in similar $2 \mathrm{DEG}^{24}$ As we can see, higher perpendicular magnetic field (or lower filling factor) is corresponding to a larger $\mathrm{g}^{*}$. Similar effect has been observed in other two-dimensional electron systems and it is attributed to

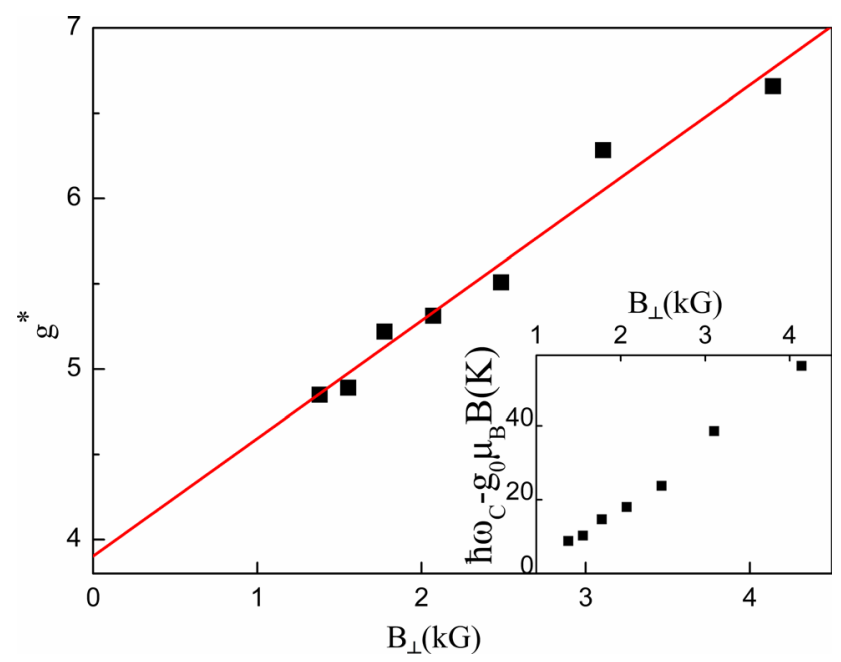

FIG. 4. Exchange-enhanced g-factor as a function of perpendicular magnetic field. The thin red line shows a linear dependence of $\mathrm{g}$-factor on $\mathrm{B}_{\perp}$, with a bare g-factor equal to 3.9. The lower-right inset: the electron-electron interaction energy, $\hbar \omega_{\mathrm{c}}-\mathrm{g}_{0} \mu \mathrm{B}$, is plotted as a function of $\mathrm{B}_{\perp}$.

many-body interactions with exchange energy. ${ }^{23,25}$ In this case, we can simply rewrite the spin-split gap as the sum of bare Zeeman energy and an exchange energy $\mathrm{E}_{\mathrm{ex}}{ }^{26}$

$$
\Delta_{\text {spin }}=\mathrm{g}^{*} \mu_{\mathrm{B}} \mathrm{B}=\hbar \omega_{\mathrm{C}}=\mathrm{g}_{0} \mu_{\mathrm{B}} \mathrm{B}+\mathrm{E}_{\mathrm{ex}} .
$$

Since $\mathrm{E}_{\mathrm{ex}}$ is proportional to $\mathrm{e}^{2} / 4 \pi \varepsilon \ell_{\mathrm{B}}$, where magnetic length $\ell_{\mathrm{B}}=\sqrt{\hbar / e B}$, we expect $\mathrm{g} *$ to be described as square-root $\mathrm{B}_{\perp}$ dependence when $\mathrm{E}_{\mathrm{ex}}$ term dominates. We plot the $\mathrm{E}_{\mathrm{ex}}=\hbar \mathrm{eB} \mathrm{B}_{\perp} / \mathrm{m}^{*}-\mathrm{g}_{0} \mu_{\mathrm{B}} \mathrm{B}_{\perp} / \cos \theta_{\mathrm{c}}$ (see inset, Fig. 4) for all the $\theta_{\mathrm{c}}$ and find an approximately linear instead of an expected square-root $\mathrm{B}_{\perp}$ dependence. This can be reconciled by considering the relevant correlation length in high Landau levels. As suggested by Leadley et al., ${ }^{27}$ here only $1 / \nu$ of the electrons contribute to the exchange energy and the correlation length should be set by the Fermi wave-vector $\mathrm{k}_{\mathrm{F}}$ rather than the magnetic length $\ell_{\mathrm{B}}$.

Finally, we focus on the small field regime where an additional type of level-crossings due to the opposite signs of Rashba and Zeeman terms is observed. As shown in Fig. 3, direct evidence of crossing can be seen in the small tilt angle regime (i.e., $1 / \cos \theta \sim 1$ ), with the crossing points marked by the downward arrows. From a crossing point tracing back to $\mathrm{B}_{\perp}=0$, the zero-field Rashba splitting gap $\Delta_{\mathrm{SO}}$ can be approximately estimated from the data. For example, the crossing around $\nu=9$ occurs at $\mathrm{B}_{\perp}=2.78 \mathrm{kG}, \mathrm{B}_{/ /}=\mathrm{B}_{\perp} \tan \theta$ $=3.43 \mathrm{kG}$. Assuming starting with zero-field, we keep the fixed perpendicular magnetic field $\mathrm{B}_{\perp}$ and sweep the in-plane magnetic field $\mathrm{B}_{/ /}$, and the transition from Rashba splitting to Zeeman splitting takes place at $\mathrm{B}_{/ /}=3.43 \mathrm{kG}$. Combining with the $\mathrm{g}^{*} \sim 5.7$ at $\mathrm{B}_{\perp}=2.78 \mathrm{kG}$ from Fig. 4, we can infer the zero-field Rashba splitting from the crossing point to be approximately, $\Delta_{\mathrm{SO}}=\mathrm{g} * \mu_{\mathrm{B}} \mathrm{B}=0.1-0.2 \mathrm{meV}$, relatively small so as to no evidence of a beating pattern null because it lays in smaller field where the $\mathrm{SdH}$ oscillations cannot be resolved clearly. This is also the reason we see over a wide field range expected for pure Zeeman splitting $\left(\mathrm{g}^{*} \mu_{\mathrm{B}} \mathrm{B} \gg \Delta_{\mathrm{SO}}\right)$ in Fig. 2.

In conclusion, we have presented a detailed and empirical Landau level spectrum in $\operatorname{In}_{0.53} \mathrm{Ga}_{0.47} \mathrm{As} / \mathrm{InP}$ quantum well structure by magneto-transport at $300 \mathrm{mK}$ in vector 
magnetic fields and analyzed the respective contributions from Rashba, bare Zeeman splitting, and the exchangecorrelation terms. An additional type of Landau levelcrossing has been observed experimentally. Utilizing inversed spin Hall effect, future experiments in generating/ detecting polarized spin current could be realized in the present $\mathrm{In}_{0.53} \mathrm{Ga}_{0.47} \mathrm{As} / \mathrm{InP}$ structure or other strongly Rashba spin-orbit coupled quantum wells.

The work at Peking University was financially supported by National Basic Research Program of China (2012CB921301 and 2014CB920901). The work at Rice was funded by DOE Grant No. DE-FG02-06ER46274 (measurement), NSF Grant No. DMR-1207562 (RRD), and Welch Foundation Grant No. C-1682 (materials).

${ }^{1}$ S. Brosig, K. Ensslin, A. G. Jansen, C. Nguyen, B. Brar, M. Thomas, and H. Kroemer, Phys. Rev. B 61, 13045 (2000).

${ }^{2}$ T. Matsuda and K. Yoh, Physica E 42, 979 (2010).

${ }^{3}$ T. Koga, J. Nitta, T. Akazaki, and H. Takayanagi, Phys. Rev. Lett. 89, 046801 (2002).

${ }^{4}$ S. Koch, R. J. Haug, K. V. Klitzing, and M. Razeghi, Phys. Rev. B 47, 4048 (1993).

${ }^{5}$ T. Kita, Y. Sato, S. Gozu, and S. Yamada, Physica B 298, 65 (2001).

${ }^{6}$ K. S. Cho, T. Y. Huang, C. P. Huang, Y. H. Chiu, C. T. Liang, and Y. F. Chen, J. Appl. Phys. 96, 7370 (2004).

${ }^{7}$ W. Desrat, F. Giazotto, V. Pellegrini, M. Governale, F. Beltram, F. Capotondi, G. Biasiol, and L. Sorba, Phys. Rev. B 71, 153314 (2005).

${ }^{8}$ B. Das, S. Datta, and R. Reifenberger, Phys. Rev. B 41, 8278 (1990).
${ }^{9}$ A. M. Gilbertson, W. R. Branford, M. Fearn, L. Buckle, P. D. Buckle, T. Ashley, and L. F. Cohen, Phys. Rev. B 79, 235333 (2009).

${ }^{10}$ C. M. Hu, J. Nitta, T. Akazaki, H. Takayanagi, J. Osaka, P. Pfeffer, and W. Zawadzki, Phys. Rev. B 60, 7736 (1999).

${ }^{11}$ S. K. Firoz Islam and T. Kanti Ghost, J. Phys.: Condens. Matter 24, 035302 (2012).

${ }^{12}$ D. Spirito, L. Di Gaspare, F. Evangelisti, A. Di Gaspare, E. Giovine, and A. Notargiacomo, Phys. Rev. B 85, 235314 (2012).

${ }^{13}$ X. Z. Liu, Y. G. Xu, G. Yu, L. M. Wei, T. Lin, S. L. Guo, J. H. Chu, W. Z. Zhou, Y. G. Zhang, and D. J. Lockwood, J. Appl. Phys. 113, 033704 (2013).

${ }^{14}$ N. Tang, B. Shen, X. W. He, K. Han, Z. J. Yang, Z. X. Qin, G. Y. Zhang, T. Lin, B. Zhu, W. Z. Zhou et al., Phys. Rev. B 76, 155303 (2007).

${ }^{15}$ B. Das, D. C. Miller, S. Datta, R. Reifenberger, W. P. Hong, P. K. Bhattacharya, J. Singh, and M. Jaffe, Phys. Rev. B 39, 1411 (1989).

${ }^{16}$ S. Q. Shen, M. Ma, X. C. Xie, and F. C. Zhang, Phys. Rev. Lett. 92, 256603 (2004).

${ }^{17}$ Z. F. Jiang, S. Q. Shen, and F. C. Zhang, Phys. Rev. B 80, 195301 (2009).

${ }^{18}$ S. Q. Shen, Y. J. Bao, M. Ma, X. C. Xie, and F. C. Zhang, Phys. Rev. B 71, 155316 (2005).

${ }^{19}$ A. Isihara and L. Smrcka, J. Phys. C: Solid State Phys. 19, 6777 (1986).

${ }^{20}$ P. T. Coleridge, Phys. Rev. B 44, 3793 (1991).

${ }^{21}$ C. Wetzel, R. Winkler, M. Drechsler, B. K. Meyer, U. Rossler, J. Scriba, J. P. Kotthaus, V. Harle, and F. Scholz, Phys. Rev. B 53, 1038 (1996).

${ }^{22}$ S. Das Sarma and F. Stern, Phys. Rev. B 32, 8442 (1985).

${ }^{23}$ R. J. Nicholas, R. J. Haug, K. v. Klitzing, and G. WeiMann, Phys. Rev. B 37, 1294 (1988).

${ }^{24}$ M. Dobers, J. P. Vieren, Y. Guldner, P. Bove, F. Omnes, and M. Razeghi, Phys. Rev. B 40, 8075 (1989).

${ }^{25}$ W. Desrat, F. Giazotto, V. Pellegrini, F. Beltram, F. Capotondi, G. Biasiol, L. Sorba, and D. K. Maude, Phys. Rev. B 69, 245324 (2004).

${ }^{26}$ J. F. Janak, Phys. Rev. 178, 1416 (1969).

${ }^{27}$ D. R. Leadley, R. J. Nicholas, J. J. Harris, and C. T. Foxon, Phys. Rev. B 58, 13036 (1998). 